Sánchez-León, Nuria.

Personal investigador en formación de carácter predoctoral, Universitat Politècnica de València, Centro de Investigación Arte y Entorno, Línea de investigación de Arte, Entorno y Sostenibilidad. orcid.org/0000-0003-3537-9156.

\title{
El papel del arte en la transformación social en contextos pioneros en el movimiento de transición a la sostenibilidad. El caso de Reino Unido.
}

\section{The role of art in social transformation in pioneering contexts in the transition movement to sustainability. The UK case.}

TIPO DE TRABAJO:

Comunicación.

PALABRAS CLAVE:

Transición a la sostenibilidad, arte transicional, arte y ecología, Reino Unido.

KEY WORDS:

Transition to sustainability, transitional art, art and ecology, United Kingdom.

\section{RESUMEN.}

La gravedad de la crisis ecológica mundial exige la participación desde todas las disciplinas para lograr un cambio cultural, sistémico y duradero hacia la sostenibilidad. En el caso del arte, la difuminación de sus límites con respecto a la autoría, el contexto y los temas en los últimos 60 años ha permitido la colaboración interdisciplinar de artistas en otros campos del saber y un replanteamiento de la función de los creadores en la sociedad. Por otra parte, los informes de la Unión Internacional de Conservación de la Naturaleza (UICN) Transición a la Sostenibilidad: hacia un mundo humanitario y diverso, señalan la necesidad de una revisión del concepto de sostenibilidad e incluso del movimiento ambientalista, para superar los retos de esta era del "Antropoceno". El concepto de transición a la sostenibilidad encuentra aplicación práctica en el movimiento de ciudades y pueblos en transición (MTC) que surge en el mundo anglosajón hace 10 años y está formado actualmente por más de 1700 iniciativas distribuidas en cerca de 50 países. Este movimiento aporta nuevas formas alternativas de actuación democrática a la organización política actual, que parten de la acción local y que recogen objetivos de descarbonización de la economía, justicia, equidad y reducción de la huella ecológica.

El objetivo de esta ponencia es estudiar las distintas formas en las que el arte ha aportado nuevos puntos de vista a los problemas ambientales, sociales o económicos fomentando prácticas de vida y actuación más sostenibles. Revisaremos a lo largo del texto los principios definidos por la autora Lucy Neal al respecto de lo que ha llamado transitional art. Siendo Reino Unido pionero en experiencias de transición, la ponencia plantea analizar una recopilación de casos anglosajones en los que se emplea el arte como estrategia de fomento de la transición de la sociedad.

\section{ABSTRACT.}

The severity of the global ecological crisis requires participation from all disciplines to achieve a cultural, systemic and lasting change towards sustainability. In the case of art, the expansion of its limits with respect to authorship, context and themes in the last 60 years has allowed the interdisciplinary collaboration of artists in other fields of knowledge and a rethinking of the role of creators in the society. On the other hand, the reports of the International Union for Conservation 
of Nature (IUCN) Transition to Sustainability: towards a humanitarian and diverse world, point to the need for a revision of the concept of sustainability and even of the environmental movement, to overcome the challenges of this "Anthropocene" era. The concept of transition to sustainability finds practical application in the movement of cities and towns in transition (TTM) that emerged in the Anglo-Saxon world 10 years ago and is currently formed by more than 1700 initiatives distributed in nearly 50 countries. This movement contributes to new alternative forms of democratic action to the current political organization, starting with local action and collecting objectives of decarbonisation of the economy, justice, equity and reduction of the ecological footprint.

The aim of this paper is to study the different ways in which art has contributed new points of view to environmental, social or economic problems by promoting more sustainable practices of life and action. We will review throughout the text the principles defined by the author Lucy Neal regarding what she has called "transitional art". As the UK is pioneer in transitional experiences, the paper proposes to analyse a collection of Anglo-Saxon cases in which art is used as a strategy to promote the transition of society.

\section{CONTENIDO.}

\section{INTRODUCCIÓN.}

La humanidad se enfrenta por primera vez en la historia a una crisis global que afecta a todos los ecosistemas del Planeta. El cambio climático y el pico del petróleo ${ }^{1}$ son los problemas más graves y urgentes ${ }^{2}$ de la era del Antropoceno ${ }^{3}$ y según los estudios científicos, ambos son inevitables. La extracción de hidrocarburos ha alcanzado su máximo ${ }^{4}$ y la inercia del cambio climático es imparable ${ }^{5} \mathrm{si}$ continuamos con este ritmo de emisiones. Dentro de este escenario se perfila el concepto de desarrollo sostenible ${ }^{6}$, respecto al cual existen numerosas definiciones y discrepancias. La Unión Internacional de Conservación de la Naturaleza señala la necesidad de una revisión del concepto ${ }^{7}$. El "desarrollo sostenible" supone a veces solo un lavado verde de los métodos existentes de producción y consumo, mientras que el "desarrollo de la sustentabilidad" se enfoca a redireccionar el desarrollo hacia una base diferente de creación de economía y sociedad, que no se base necesariamente en el crecimiento económico y el consumo sino en mejorar la calidad de vida de todos.

Frente a la situación climática de crisis, el poder tecno-científico se afana en ignorar los conocidos límites de los recursos ${ }^{8}$ aun cuando ya no puede evitar el inminente colapso energético. La visión cartesiana, del problema ecológico se muestra insuficiente ante una crisis de características sistémicas sustentada por el propio orden cultural, económico y social. Se imponen medidas que trasciendan el conocimiento disciplinario totalizador y que abarquen la complejidad del problema ${ }^{9}$. Esta transición hacia una nueva cultura ecológica ${ }^{10}$ debe incluir todas las disciplinas y todos los saberes, ser ordenada y gradual y tender hacia una nueva sociedad con un menor consumo de recursos ${ }^{11}$.

\footnotetext{
${ }^{1}$ O cenit del petróleo barato, supone el inicio del descenso de la extracción, anunciado ya por el científico M. King Hubbert en 1956.

2 BRANGWYN, B., HOPKINS, R., MOOSER, R. (trad) y MONZÓN, Á. (trad), 2010. Compendio de iniciativas de transición. Cómo convertirse en una población, ciudad, distrito, pueblo comunidad e incluso isla en transición. [en línea]. Disponible en: http://movimientotransicion.pbworks.com/w/page/23759307/Compendio sobre la Transicion.

${ }^{3}$ Término definido por P. J. Crutzen en el 2000, CRUTZEN, P.J. y STOERMER, E.F., 2000. The «Anthropocene». Global Change Newsletter [en línea], vol. 41, no. 41, pp. 17-18. Disponible en:

http://www.igbp.net/publications/globalchangemagazine/globalchangemagazine/globalchangenewslettersno4159.5.5831d9ad13275d51c09 8000309.html.

${ }_{4}^{4}$ TURIEL, A., 2017. The crash oil. 1 marzo 2017 [en línea]. [Consulta: 13 marzo 2017]. Disponible en: http://crashoil.blogspot.com.es/

${ }^{5}$ BERGER, A. y LOUTRE, M.F., 2002. An Exceptionally Long Interglacial Ahead? Science's compass [en línea], vol. 297, no. August, pp. 1287-1288. Disponible en: ftp://ftp.soest.hawaii.edu/engels/Stanley/Textbook_update/Science_297/Berger-02.pdf.

${ }^{6}$ descrito en 1987 en la Convención de Naciones Unidas para el Medio Ambiente y el Desarrollo y "el desarrollo que satisface las necesidades del presente sin comprometer la habilidad de las futuras generaciones de satisfacer sus propias necesidades" DEMOS, T.J., 2013. The Politics of Sustainability: Art and Ecology. ... Nature: Art and Architecture for a ... [en línea], pp. 16-30. Disponible en: http://sustainabilityparadox.commons.gc.cuny.edu/files/2010/09/Demos-Politics-of-Sustainability.pdf. p.18

${ }^{7}$ W.M. ADAMS Y S.J. JEANRENAUD (UICN), 2008. Transición a la Sostenibilidad : hacia un mundo humanitario y diverso. Gland, Suiza.

${ }^{8}$ MEADOWS, D., MEADOWS, D., RANDERS, J. y BEHRENS III, W.W., 1972. Los límites del crecimiento. S.I.: s.n.

${ }^{9}$ MORIN, E., 2012. Edgar Morin El padre del pensamiento complejo. [en línea]. [Consulta: 3 agosto 2016]. Disponible en: hhttp://www.edgarmorin.org/que-es-transdisciplinariedad.html.

${ }^{10}$ GUATTARI, F., 1996. Las tres ecologías. Valencia: Pretextos.

11 "el decrecimiento es el único debate que tiene sentido" dice A. Turiel en FORO TRANSICIONES. Debate Hills Group. Madrid el 20 de febrero 2017.
} 
En esta línea de adaptación al cambio climático y desarrollo de resiliencia ante el colapso energético, Reino Unido ha sido pionero en dos sentidos. El primero con la experiencia del movimiento de ciudades y pueblos en transición surgido en el $2005^{12}$. Está formado actualmente por más de 1.107 iniciativas en más de 43 países (2013) según el registro llevado a cabo por la web transitionnetwork.org. Este movimiento se cimenta sobre la idea de disminuir el consumo de energía y recursos de la comunidad para ser más resilientes y afrontar con mayor éxito los dos problemas fundamentales ya mencionados. Abarca una tipología de iniciativas muy variada como eco aldeas, huertos urbanos, granjas de permacultura, espacios ocupados, monedas locales o cooperativas entre otros.

En segundo lugar, Reino Unido cuenta con una tradición de artistas que vienen trabajando desde los años 70 en la relación del arte con el entorno natural desde el land art, el environmental art, el eco-art y el site specific tales como: Hamish Fulton, Richard Long, David Nash, Andy Goldsworthy, la plataforma de artistas Platform o Tim Collins y Reiko Goto residentes en Reino Unido.

La larga trayectoria anglosajona en cuanto a la transición a la sostenibilidad y la relación arte-ecología, motivó una estancia de investigación de seis meses con base en Aberdeen (Escocia). Durante la misma se exploraron mediante metodologías cualitativas como la investigación Acción Participativa (IAP) y las entrevistas, experiencias de arte relacionado con la transición a la sostenibilidad. A continuación se expone parte de la investigación y se presentarán las conclusiones iniciales.

\section{DESARROLLO.}

Una vez señalada la urgencia de la crisis ambiental y la exigencia de afrontarla transdisciplinarmente, queda patente la necesidad de actuación también desde el campo artístico, en el fomento de otras prácticas de vida más sustentables. Artistas como Mierle Ukeles con su "Manifiesto" de 1969 o Joseph Beuys, autor del concepto "escultura social" han contribuido en los últimos 60 años a la expansión del arte hacia la vida diaria, con un enfoque de responsabilidad eco-social en el caso de Beuys, que reside en los fundamentos de lo que la artista británica Lucy Neal ha denominado, de modo pionero, como transitional art. De este modo, el papel del arte en la transición a la sostenibilidad implica una idea expandida del arte, un arte al servicio de la vida ${ }^{13}$ en la que las prácticas vitales diarias conforman la escultura común que construye la sociedad. El artista cede el protagonismo creador en favor de la coautoría y colaboración entre artistas-científicos- expertos y público. El transitional art comparte características con el llamado arte comprometido social o ecológicamente, arte público de nuevo género o práctica dialógica. Ahora bien, siguiendo a Neal, en este caso los temas a tratar se corresponden con las grandes narrativas de la transición a la sostenibilidad ya mencionadas (2015). Así pues los principios del arte transicional señalados por Lucy Neal ${ }^{14}$ son los siguientes:

1. Intención: crea la condiciones para el cambio sin necesidad de un plan previo Intención: crea la condiciones para el cambio sin necesidad de un plan previo

2. Ignición: actúa, transforma las ideas de la imaginación a la realidad

3. Marco: conforma la estructura y la estética de un proyecto y crea un contexto o narrativa para explorar.

4. Trabajar con la comunidad: despierta el coraje necesario para encarar los retos globales que no podemos afrontar individualmente y construye resiliencia dando esperanza a los individuos en la comunidad.

5. Mediación: hace que las cosas fluyan sin esfuerzo, cruzar fronteras entre disciplinas y sectores, crear redes y abrir puertas y caminos.

6. Mantener un espacio: focaliza las actitudes y energías del grupo, da seguridad y materializa la unión entre sus miembros.

7. Conectar: cuando se trascienden los límites del individuo y se construye una historia compartida, se crea un espacio para una narrativa holística y coherente que conecte las grandes problemáticas ambientales con una narrativa local de comunidad y cambio positivo.

8. Trabajar desde lo común: frente a una cultura de la diferencia y la confrontación se centra en lo común y el diálogo para entender y compartir las sensaciones del otro.

\footnotetext{
${ }^{12}$ HAXELTINE, A. y SEYFANG, G., 2009. Transitions for the People: Theory and Practice of 'Transition ' and 'Resilience ' in the UK ' $s$ Transition Movement. Transition [en línea]. Norwich: 1st European Conference on Sustainability Transitions, 134. Disponible en: http://scholar.google.com/scholar?hl=en\&btnG=Search\&q=intitle:Transitions+for+the+People:+Theory+and+Practice+of+\&\#39; Transition\& $\% 2339 ;$ and +\&\%2339; Resilience \&\%2339;in+the+UK\&\%2339;s+Transition+Movement $\% 230$

${ }_{13}^{13}$ NEAL, L., 2015. Playing for Time. Making art as if the world mattered. 1. Londres: Oberon books. ISBN 9781783191864. p.14

${ }^{14}$ Ibid pp. 81-93
} 
9. Colaborar: se trabaja con apertura en equipo y en un equilibrio complejo de tensiones para lograr un mejor resultado que a nivel individual.

10. Cambio: se puede fomentar desde la intención con la que comienza el proyecto y compartir para crear una gran narrativa del cambio.

Neal recopila en el libro Playing for time. Making art as if the world mattered hasta 69 proyectos que responden a todos o gran parte de estos principios. Es especialmente en el último punto, la intención de cambio, donde surgen discrepancias con otros artistas que también trabajan con un enfoque ecológico. De una parte algunos artistas han aportado soluciones y propuestas de cambio más o menos directas, como la biorremediación en el caso de Patricia Johansson en Fair Park Lagoon (1981-86) o las descritas por Neal en dicho compendio. De otra, artistas pioneros del environmental art como Newton y Helen M. Harrison declaran no tener la intención de cambiar las ideas del público, ni convencerles de cambiar su comportamiento ${ }^{15}$. Asimismo los artistas Reiko Goto y Tim Collins declaran que el arte no se ocupa de dar respuestas correctas, ni soluciones definitivas a la urgencia de la crisis ecológico-social ${ }^{16}$. Su obra se dirige hacía cambiar las preguntas que el público se hace respecto al medio ambiente, a crear imágenes que resuenen en la consciencia, a inspirar un cuestionamiento, a mostrar otras alternativas y futuros, posibles ahora solo en nuestra imaginación, pero más cercanos una vez imaginados. Observamos por tanto, que existe una gradación de posturas ante la necesaria transición, desde obras más prácticas y directas hasta otras sugerentes e inspiradoras. Desde esta investigación se examinan, a la luz de estos principios, dos proyectos artísticos con diferente rango en esta gradación y se estudia el papel del artista en los mismos. A partir de los resultados de estos proyectos se explora el papel del arte en la transformación social. La investigación se ha llevado a cabo mediante metodologías cualitativas como la Investigación Acción Participativa, formando parte de las actividades de estos proyectos y también realizando entrevistas tanto a los artistas como al público participante.

\section{The Stoves: proyectos We live with water y Nith Raid.}

En primer lugar, se examinan los proyectos We live with water y Nith Raid de The Stoves, en Dumfries, Escocia. Dumfries es una población que vive sin establecer ninguna relación con el río que la atraviesa, el Nith. The Stove es un colectivo de artistas y habitantes que se proponen intervenir y dinamizar el espacio público de la población creando momentos y espacios de relación. Como en tantas poblaciones escocesas, el centro de la ciudad se ha desplazado a los centros comerciales de la periferia, mucho más impersonales, dejando el centro de la ciudad inanimado. Paralelamente el cambio climático ha acentuado las crecidas anuales del río. El Ayuntamiento se plantea afrontarlas construyendo una barrera de 3 metros de altura ${ }^{17}$. Sin embargo el proyecto que plantean los artistas en We live with water ${ }^{18}$ es una idea muy distinta del futuro de Dumfries en 2065: en lugar de confrontar las fuerzas del río, proponen, en un proyecto escrito de ocho páginas, facilitarle el paso para su crecida anual, contener el desarrollo urbano e incluso retirar algunos negocios y aparcamientos que ocupan la orilla de inundación del Nith (en la ilustración 1, zona 5) Lejos de ser una retirada penosa, se propone hacer de ello incluso una celebración que reúna a la población en torno al río. Para ello, todos los 3 de septiembre desde hace cuatro años se organiza la Nith Raid, una carrera festiva en barco a lo largo del Nith acompañada de talleres eco-artísticos, música y comida, que celebra la presencia del río, e invita a toda la población a establecer una nueva relación con él. Es un giro de actitud hacia una resiliencia duradera, que implica a largo plazo mucho menos esfuerzo que la lucha constante. La función del artista en este caso es la de imaginar y plantear otros futuros y relaciones posibles alternativas a la tendencia de solucionar adversidades climatológicas a base de inversión tecnológica e ingeniería.

\footnotetext{
${ }^{15}$ Helen M. Harrison, 2008 en GOTO COLLINS, R., 2012. Ecology and environmental art in public place talking tree: won ' $t$ you take a minute and listen to the plight of nature? [en línea]. S.I.: Robert Gordon University. [Consulta: 28 octubre 2015]. Disponible en: http://collinsandgoto.com/wp-content/uploads/2012/04/Reiko-PHD-Thesis-Reiko-Goto.pdf.pp.138-139 ${ }_{16}^{16}$ (comunicación personal, 14 de octubre de 2016)

${ }^{17}$ En este sentido el artista D. Haley apunta que la sociedad está demasiado acostumbrada a esa visión masculina de dominación sobre la naturaleza. CIWEM, T.C.I. of W. and E.M., 2012. WE ASSERT! WE ASSERT ! The Manifesto of CIWEM's Arts and Environment Network (AEN). [en línea]. Londres, Reino Unido: Disponible en: http://www.ciwem.org/wp-content/uploads/2016/03/WE-ASSERTFINAL.compressed.pdf.

${ }^{18}$ ANDERSON, K., FOSTER, K., PACHECO, R., JONES, A., SLATER, D., BONAVENTURA, M., MCQUEEN, L., BAKER, M., ZYGADLO, M., GOTT, I., WHITE, S., SMITH, M., SOUTAR, L., DEWAR, R., POWELL, L. y WHEELER, K., 2015. We live with water. 2065: life today in Dumfries River Town. [en línea]. Dumfries: Disponible en: http://www.thestove.org/we-live-withwater/?doing_wp_cron=1489075817.1538279056549072265625.
} 


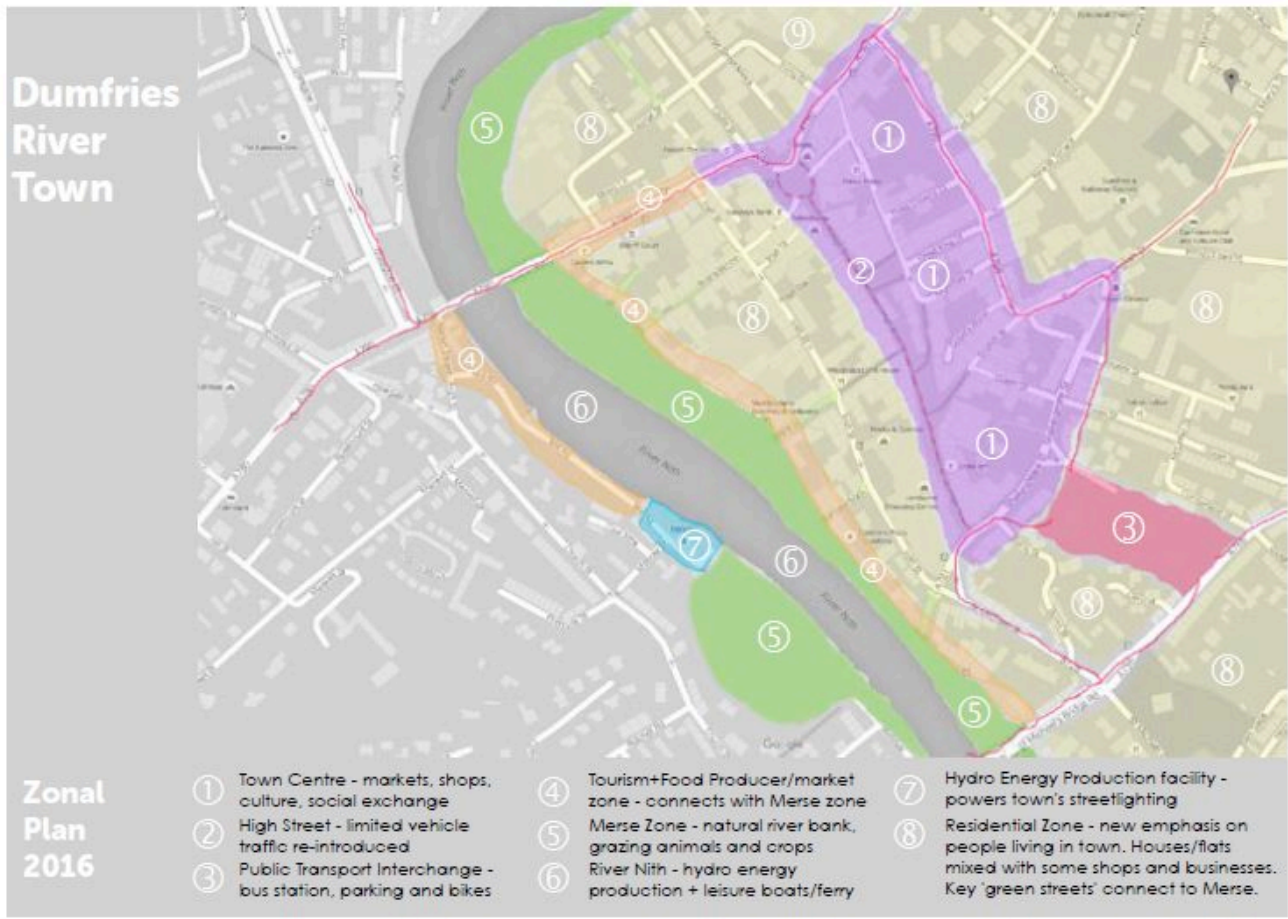

Ilustración 1. Nuevo planeamiento urbano propuesto por el proyecto We live with water.

Si analizamos el proyecto desde los principios del transitional art, se detecta que existe una intención que llama a la actuación y transforma las perspectivas de inundación del futuro de Dumfries en una nueva realidad. El marco del proyecto es el presente y futuro del río y se trabaja con la comunidad en la ceremonia festiva. Los artistas actúan de facilitadores de una nueva relación con el agua y con el espacio del río. Mediante la implantación de una nueva costumbre cada septiembre, pretenden conectar a la población con el torrente creando una nueva tradición compartida. Trabajan con el espacio común para unir, aunque para ello propongan una rendición que ceda el área que le corresponde a la riada. La colaboración en la Nith Raid es fundamental y numerosos voluntarios toman parte en la organización. No obstante, el cambio de actitud que proyectan en We live with water es solo una invitación sutil a imaginar, aunque se hizo llegar al Primer Ministro Richard Arkless en su visita a la ciudad. Sin embargo, ninguno de los artistas entrevistados de The Stove (Robbie Coleman, Jan Hoggarth y Matt Baker) considera su obra como transitional art ${ }^{19}$ (comunicación personal, 18 de julio de 2016) Ni siquiera se plantean objetivos de transición a la sostenibilidad y apenas Matt Baker menciona la justicia ambiental. Su verdadero objetivo es dinamizar el espacio público en Dumfries. La propuesta de We live with water no ha dado como resultados más allá de una anécdota política que no fue tenida en cuenta en el parlamento inglés. Sin embargo, la Nith raid, que es un proyecto mucho más participativo, reúne cada año a más participantes alrededor de la rivera en al menos, ese momento del año. Poco a poco esta celebración participativa está tejiendo una nueva relación entre la población y el agua.
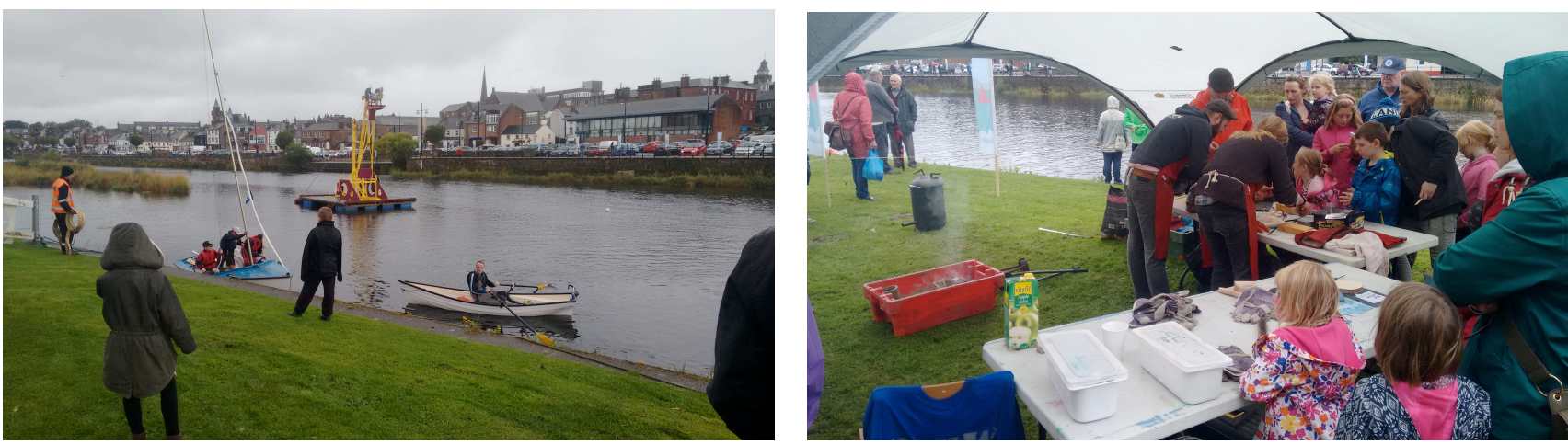

llustraciones 2 y 3. La carrera Nith Raid en septiembre 2016. En la orilla más lejana se observa el aparcamiento y la vía que ocupan la orilla de inundación del río Nith. Fotos de la autora.

${ }^{19}$ (comunicación personal, 12 de diciembre de 2016). 


\section{Dundee Urban Orchard.}

El segundo proyecto estudiado es Dundee Urban Orchard (DUO) de los artistas Sarah Gittins y Jonathan Baxter. En el contexto de la ciudad de Dundee, la quinta más poblada en Escocia, pero con un alarmante nivel de desempleo, su propuesta busca responder la narrativa capitalista, en concreto el sistema industrial de producción de alimentos, la pobreza alimentaria, la falta de visión social, y medioambiental. El proyecto artístico apoya a individuos, grupos comunitarios y organizaciones culturales a plantar y cuidar huertos pequeños ${ }^{20}$ y trabajar con la ciudad entera en su conjunto como la metáfora de un gran huerto. Surge del esfuerzo por imaginar un nuevo Dundee de un grupo de lectura organizado en la biblioteca municipal. ¿Cómo podría ser Dundee? ¿Cómo sería si los patios y espacios ajardinados en desuso fueran huertos? ¿Qué podemos aprender de los huertos como modelo de desarrollo urbano sostenible? La respuesta del grupo de lectura está inspirada por el concepto de escultura social de Joseph Beuys y la defensa de la biodiversidad de Vandana Shiva ${ }^{21}$. Es materializada por los artistas y habitantes en un conjunto de 25 huertos comunitarios urbanos dispersos por la ciudad. Cada uno de ellos con particularidades y colectivos responsables diferentes, desde bibliotecarios a voluntarios de la iglesia, alumnado del colegio o individuos en sus jardines privados.
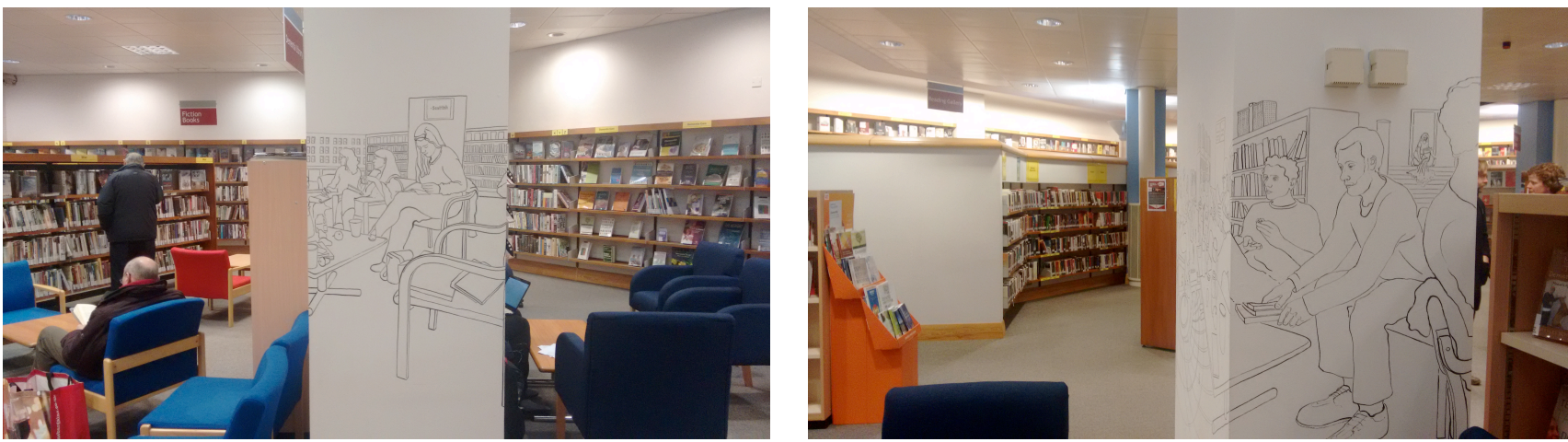

llustraciones 4 y 5 . La traza plasmada en la biblioteca del grupo de lectura debatiendo como podría ser Dundee. Fotos de la autora.

Respecto a los principios ya mencionados, en este proyecto la intención de los artistas parte de sacar el arte del "cubo blanco", materializar las ideas y deseos de la población local sin imponer su criterio, implicar a los habitantes en los espacios públicos, y trabajar con ellos en problemáticas que les interesan como la producción de alimentos. El proyecto artístico pretende aumentar la biodiversidad y la resiliencia por lo que engarza con los objetivos de la transición a la sostenibilidad pero "sin convertirlo en una lección" ${ }^{22}$. El marco abarca toda la ciudad aprovechando los espacios ajardinados que ya existen. Se trabaja con la comunidad que, al sentirse dentro de un proyecto vivo y no aislado, encuentran apoyo en otros miembros. El artista en este caso ha mediado con las comunidades, organismos e individuos invitándoles a crear su propio huerto y facilitando el proceso. El espacio está disperso y en algunos casos es privado pero todo interesado encuentra un lugar en el que aportar. Conecta individuos e historias que de otro modo no tendrían relación dando fuerza a una iniciativa de cambio positivo.

Lo común en este caso son la alimentación, el goce de disfrutar cultivando y los espacios públicos como la biblioteca. La colaboración es un factor esencial. Finalmente, el cambio en los espacios es mucho más patente en alguna iniciativa que en otra. Proyectos exitosos como el huerto del Centro MAXwell permiten a cuatro grupos de escolares una experiencia directa de cultivo que antes era inexistente. Pero se ignora el cambio en sus actitudes con respecto al consumo o a la relación con el espacio público. Los artistas no han realizado ninguna medición.

En este proyecto se supera la mera representación que realiza The Stove en We live with water y transforma las ideas de un nuevo Dundee $^{23}$ en realidades mediante la actividad performativa artística. En ese sentido es un proyecto que se acerca más a la posición del transitional art. Por el contrario, el proyecto We live with water y la propia Nith Raid corresponden a una línea de acción que propone nuevos futuros posibles pero no cambia realmente el planeamiento urbano.

\footnotetext{
${ }^{20}$ BAXTER, J. y GITTINS, S., 2013. Dundee Urban Orchard. [en línea]. [Consulta: 12 marzo 2017]. Disponible en: https://dundeeurbanorchard.net/.

${ }^{21}$ Ibid.

22 (comunicación personal 12 de diciembre de 2016, 1:03')

${ }^{23}$ Traducción de la autora "Y no solo representar un nuevo Dundee que es lo que la mayoría de los artistas hace", en palabras de Jonathan Baxter (comunicación personal, 12 de diciembre de 2016, 30')
} 

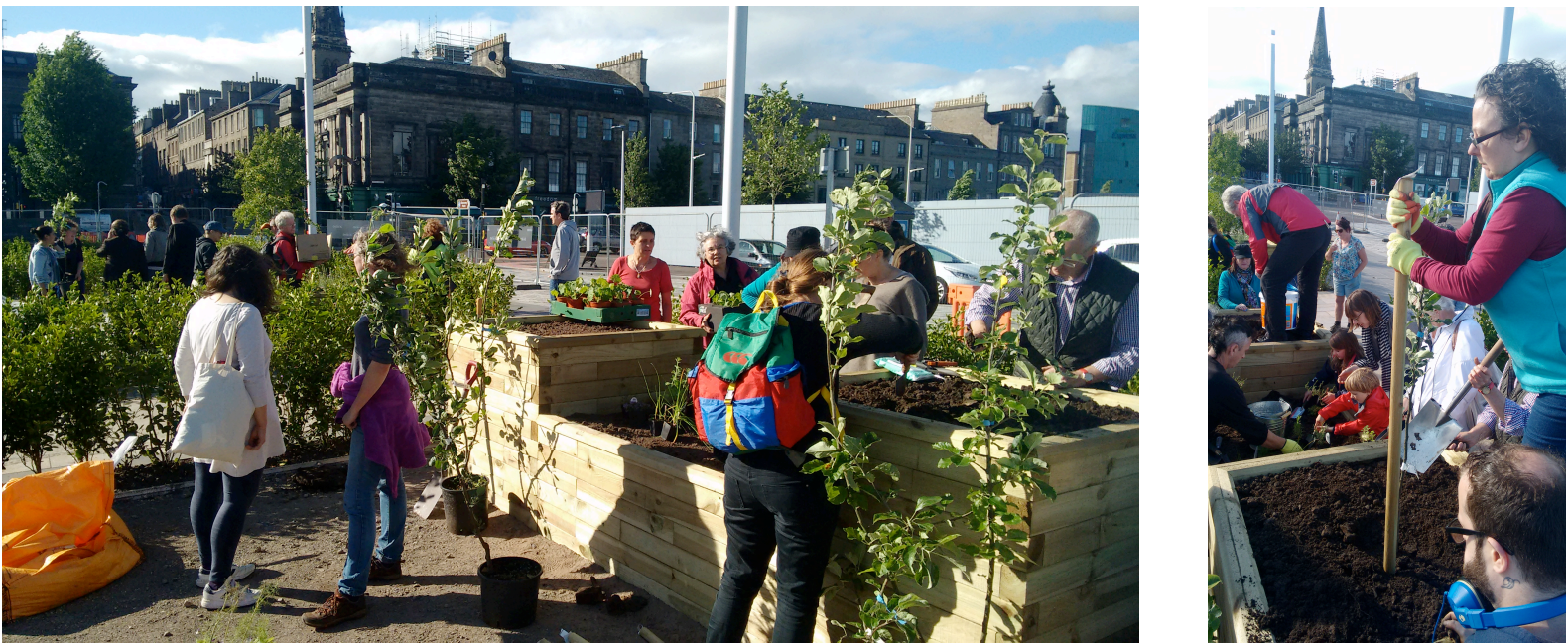

Ilustraciones 5 y 6 . Huerto de frutales y aromáticas, Slessor Gardens en la nueva ampliación del puerto de Dundee. Foto de la autora

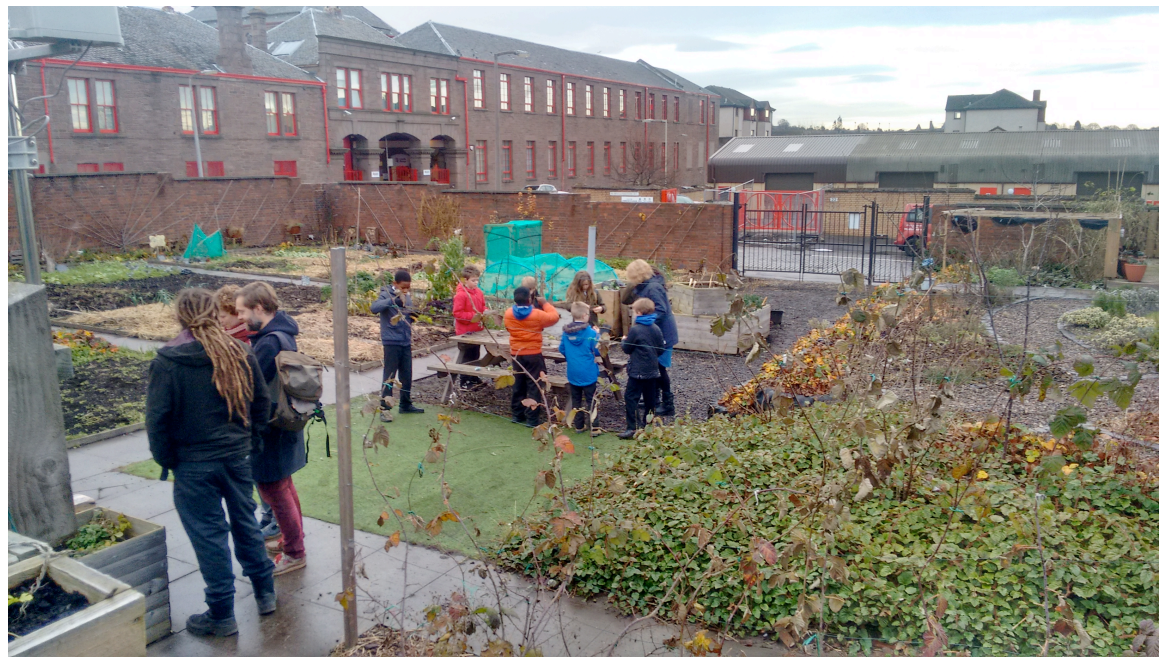

Ilustración 7. Centro comunitario MAXwell: huerto detrás de la iglesia con niños participando. Foto de la autora

\section{CONCLUSIONES.}

La experiencia de estancia de investigación en Reino Unido ha supuesto una fructífera etapa en la investigación del papel del arte en la transformación social frente a la crisis ecológica global. Nos ha permitido entrar en contacto con el transitional art y comprobar su aplicación sobre el terreno. Como primera conclusión, apuntamos que los diez principios señalados por Lucy Neal no parecen determinantes a la hora de establecer sí un proyecto fomenta la transición a la sostenibilidad en mayor o menor medida. Los temas que forman la gran narrativa de la transición son muy amplios y numerosos proyectos se pueden incluir dentro de esta temática. Así mismo, los principios son muy genéricos como para ser definitorios y algunos en particular incluso obvios. En todo caso, el conjunto parece señalar que el transitional art está más focalizado al cambio y a la obtención de resultados, lo cual desafía la opinión de otros artistas que también contribuyen a la transformación a una sociedad más sustentable.

Por otro lado, los resultados de los proyectos analizados parecen apuntar a la participación y la colaboración de los habitantes, como un factor clave para el éxito de la transformación social propuesta por los artistas. Sin duda en ambos casos estudiados ha habido una transformación en las vidas de las personas involucradas en el caso de los proyectos de Dundee Urban Orchard de un modo más constante y en el caso de The Stove, puntual pero creciente. También es reseñable que en ninguno de los dos casos los proyectos son considerados por sus creadores como transitional art, bien porqué ignoran el término como en el primer caso o bien porque evitan etiquetas. 
El papel del arte en la transformación social en contextos pioneros en el movimiento de transición a la sostenibilidad. El caso de Reino Unido. III CONGRESO INTERNACIONAL DE INVESTIGACIÓN EN ARTES VISUALES: ANIAV 2017: GLOCAL [codificar, mediar, transformar, vivir] http://dx.doi.org/10.4995/ANIAV.2017.5806

Desde un punto de vista práctico podemos entender las actuaciones de estos artistas cómo proyectos piloto, maquetas de otras realidades que podrían funcionar a largo plazo en estos contextos. Donde la ciencia solo aporta modelizaciones, estos artistas están poniendo en práctica las ideas de los habitantes demostrando día a día que otras prácticas de vida podrían ser posibles.

\section{FUENTES REFERENCIALES.}

ANDERSON, K., FOSTER, K., PACHECO, R., JONES, A., SLATER, D., BONAVENTURA, M., MCQUEEN, L., BAKER, M., ZYGADLO, M., GOTT, I., WHITE, S., SMITH, M., SOUTAR, L., DEWAR, R., POWELL, L. y WHEELER, K., 2015. We live with water. 2065: life today in Dumfries River Town. [en línea]. Dumfries: Disponible en: http://www.thestove.org/we-live-withwater/?doing_wp_cron=1489075817.1538279056549072265625.

BAXTER, J. y GITTINS, S., 2013. Dundee Urban Orchard. [en línea]. [Consulta: 12 marzo 2017]. Disponible en: https://dundeeurbanorchard.net/.

BERGER, A. y LOUTRE, M.F., 2002. An Exceptionally Long Interglacial Ahead? Science's compass [en línea], vol. 297, no. August, pp. 1287-1288. Disponible en: ftp://ftp.soest.hawaii.edu/engels/Stanley/Textbook_update/Science_297/Berger-02.pdf.

BRANGWYN, B., HOPKINS, R., MOOSER, R. (trad) y MONZÓN, Á. (trad), 2010. Compendio de iniciativas de transición. Cómo convertirse en una población, ciudad, distrito, pueblo comunidad e incluso isla en transición. [en línea]. Disponible en:

http://movimientotransicion.pbworks.com/w/page/23759307/Compendio sobre la Transicion.

CIWEM, T.C.I. of W. and E.M., 2012. WE ASSERT ! WE ASSERT ! The Manifesto of CIWEM's Arts and Environment Network (AEN). [en línea]. Londres, Reino Unido: Disponible en: http://www.ciwem.org/wp-content/uploads/2016/03/WE-ASSERT-FINAL.compressed.pdf.

CRUTZEN, P.J. y STOERMER, E.F., 2000. The «Anthropocene». Global Change Newsletter [en línea], vol. 41, no. 41, Disponible en: http://www.igbp.net/publications/globalchangemagazine/globalchangemagazine/globalchangenewslettersno4159.5.5831d9ad13275 d51c098000309.html.

DEMOS, T.J., 2013. The Politics of Sustainability: Art and Ecology. Radical Nature: Art and Architecture for a Changing Planet 19692009 [en línea], pp. 16-30. Disponible en: http://sustainabilityparadox.commons.gc.cuny.edu/files/2010/09/Demos-Politics-ofSustainability.pdf.

GOTO COLLINS, R., 2012. Ecology and environmental art in public place talking tree: won ' $t$ you take a minute and listen to the plight of nature? [en línea]. S.I.: Robert Gordon University. [Consulta: 28 octubre 2015]. Disponible en: http://collinsandgoto.com/wpcontent/uploads/2012/04/Reiko-PHD-Thesis-Reiko-Goto.pdf.

GUATTARI, F., 1996. Las tres ecologías. Valencia: Pretextos.

HAXELTINE, A. y SEYFANG, G., 2009. Transitions for the People : Theory and Practice of Transition ' and Resilience ' in the UK' $s$ Transition Movement. Transition [en línea]. Norwich: 1st European Conference on Sustainability Transitions, 134. Disponible en: http://scholar.google.com/scholar?hl=en\&btnG=Search\&q=intitle:Transitions+for+the+People:+Theory+and+Practice+of+\&\#39;Transit ion\&\%2339; and+\&\%2339; Resilience\&\%2339;in+the+UK\&\%2339;s+Transition+Movement\%230.

MEADOWS, D., MEADOWS, D., RANDERS, J. y BEHRENS III, W.W., 1972. Los límites del crecimiento. S.I.: s.n.

MORIN, E., 2012. Edgar Morin El padre del pensamiento complejo. [en línea]. [Consulta: 3 agosto 2016]. Disponible en: hhttp://www.edgarmorin.org/que-es-transdisciplinariedad.html.

NEAL, L., 2015. Playing for Time. Making art as if the world mattered. 1. Londres: Oberon books. ISBN 9781783191864.

TURIEL, A., GONZÁlEZ, L., RIECHMANN, J., SERRANO, A., PRIETO, P., DE CASTRO, C., NIETO, J. y GARCÍA CASALS, X., 2017. FORO TRANSICIONES. Debate Hills Group. Madrid: s.n.,

TURIEL, A., 2017. The crash oil. 1 marzo 2017 [en línea]. [Consulta: 13 marzo 2017]. Disponible en: http://crashoil.blogspot.com.es/ 